\title{
Spatially- and Temporally-resolved Multi-parameter Interferometric Rayleigh Scattering
}

\author{
Daniel Bivolaru* and Andrew. D. Cutler ${ }^{\dagger}$ \\ The George Washington University, Newport News, VA, 23602 \\ and \\ Paul M. Danehy* \\ NASA Langley Research Center, Hampton VA, 23681-2199
}

\begin{abstract}
A novel approach to simultaneously measure the translational temperature, bulk velocity, and density in gases by collecting, referencing, and analyzing nanosecond time-scale Rayleigh scattered light from molecules is described. A narrow-band pulsed laser source is used to probe two largely separated measurement locations, one of which is used for reference. The elastically scattered photons containing information from both measurement locations are collected at the same time and analyzed spectrally using a planar Fabry - Perot interferometer. A practical means of referencing the measurement of velocity using the laser frequency, and the density and temperature using the information from the reference measurement location maintained at constant properties is described. To demonstrate the technique single-shot spectra of elastic scattered light are obtained in a near zero velocity $\mathrm{H}_{2^{-}}$ air Hencken burner flame and simultaneously in an $\mathrm{N}_{2}$-filled gas cell. A simplified Gaussian distribution model to the scattered light spectra is used to obtain the flame properties. Corrections to this model are applied at lower gas temperatures when the simplified Gaussian approximation is no longer suitable. The near-zero measured velocity as a function of the measured flame temperature, and a comparison of the measured flame density and temperature with the perfect gas law are presented.
\end{abstract}

\section{Introduction}

$\mathrm{M}$ OLECULAR-BASED diagnostics techniques capable of obtaining simultaneous multiple fluid properties such as temperature, density and velocity are critically important for characterizing the flows within airbreathing engines, such as scramjet engines and scramjet - turbine combined cycle engines for hypervelocity vehicles. At the other end of the velocity range, such techniques are needed to help in the development of the next generation of subsonic, low noise aircraft. Statistical correlations between these properties will lead to a more detailed understanding of the complex flow behavior as well as aid in the development of new turbulence models for scramjet engine flow path designs, ${ }^{1,23}$ and more accurate aircraft noise prediction tools. ${ }^{4}$ Required are instantaneous and simultaneous measurements of temperature, velocity, density, pressure, and chemical composition at multiple points in a gas when the spatial (hundreds of microns or less) and temporal (hundreds of nanoseconds or less) scales of the turbulent fluctuations are resolved. Specifically of interest are the mean properties and their turbulent variances and co-variances as well as the probability density functions. To date, diagnostics techniques to meet all these requirements on a single platform do not exist, but many linear and non-linear optical techniques are available for partial achievement of this goal. ${ }^{5,6}$ One of these linear techniques, the Interferometric Rayleigh Scattering

\footnotetext{
*Research Scientist, School of Engineering and Applied Sciences, Mechanical and Aerospace Engineering Department, in residence at NASA LaRC/ASOMB, MS 493, Senior AIAA Member.

${ }^{\dagger}$ Professor, School of Engineering and Applied Sciences, Mechanical and Aerospace Engineering Department, 100 Old Oyster Point Road, Suite 200, AIAA Associate Fellow.

*Research Scientist, Advanced Sensing and Optical Measurement Branch (ASOMB), MS 493, AIAA Associate Fellow.
} 
technique (IRS), has been used for low- as well as for high-speed supersonic/hypersonic non-reacting and combusting flows yielding temporally and spatially resolved simultaneous measurements of two-components of bulk velocity. ${ }^{7}$ Recent efforts have attempted to obtain the ro-vibrational temperature, and the species content of $\mathrm{N}_{2}$ and $\mathrm{O}_{2}$ from measurements with $\mathrm{CARS}^{8}$ (coherent anti-Stokes Raman spectroscopy), simultaneously with velocity with IRS. ${ }^{9,10}$ However, this work in progress needs improvements in instrument precision, as well as additional instantaneous measurements, the gas density and a measurement of translational temperature.

In response to these research needs, in this paper we introduce a novel multi-parameter IRS technique for simultaneously obtaining the translational temperature, the bulk velocity, and the density in gases at elevated temperatures in a non-intrusive manner. A laboratory demonstration measuring these properties in a $\mathrm{H}_{2}$ - air combustion flame (Hencken burner), relative to simultaneous measurements in a reference $\mathrm{N}_{2}$ - filled cell, is described. The measurements are compared with the adiabatic flame theory predictions and the applicability of this method to turbulent flows is discussed in this paper.

\section{Principle}

The interferometric Rayleigh scattering (IRS) technique uses a narrow-band polarized laser source of wavelength $\lambda_{0}$ for probing gas molecules situated at a given measurement volume through photon scattering. Both elastic Rayleigh and inelastic Raman scattering of photons occurs. The inelastic scattering arises from changes in the rotational and vibrational states of the molecules during the scattering process. This process is weak and occurs in a different wavelength band than the laser wavelength. The Rayleigh scattering process is directly related to the spatio-temporal motion of the gas molecules and does not change the internal energy of the molecules. It is orders of magnitude stronger and occurs near the laser wavelength. The number of photons scattered are proportional to the incident laser energy. Receiving optics directly collect the scattered light (near $\lambda_{0}$ ) in a small solid angle, optically compare it with laser light for frequency reference, and analyze it spectrally with a high-resolution Fabry-Perot interferometer and a CCD camera detector. ${ }^{11,12}$ The details of the spectral structure are related to the state of collisions between gas molecules traditionally divided in two regimes: hydrodynamic or collision dominated, and kinetic or collisionless regime (Rayleigh regime), without a clear border between them. The shape of the spectra is related to a non-dimensional ordering parameter $y=\alpha / K a$, where $\alpha=p / \mu$ is the collision frequency ( $p$ is the gas pressure and $\mu$ is the shear viscosity) and $K a$ is the acoustic spatial frequency ( $K$ is the magnitude of the resultant wave vector $\mathbf{K}$ and $a$ is the speed of sound). For "s" type scattering, the angle between the electric field vector $\mathbf{E}$ of the incident light of wave vector $\mathbf{k}_{0}$ (vertically polarized) and the direction of the scattered light of wave vector $\mathbf{k}_{\mathrm{s}}$ (horizontal direction) is $\beta=90^{\circ}$. We denote the scattering angle $\theta$, as the angle between the direction of $\mathbf{k}_{0}$ vector and the direction of $\mathbf{k}_{\mathrm{s}}$ vector along the optical axis of the receiving optics. With these notations the resultant wave vector is $\mathbf{K}=\mathbf{k}_{\mathrm{s}}-\mathbf{k}_{0}$. Assuming that the wave vectors magnitudes are about the same $\left|\mathbf{k}_{\mathrm{s}}\right| \approx\left|\mathbf{k}_{0}\right|$, the magnitude of the resultant wave vector is

$$
K=2 k_{0} \sin (\theta / 2)
$$

where $k_{0}=\left|\mathbf{k}_{0}\right|=2 \pi / \lambda_{0}$. For very low density gases $y$ is negligibly small and the Rayleigh spectra is approximated with a Gaussian shaped spectra. For moderate density gases, when collisions between molecules start to dominate $y$ $\sim 1$ ), the spectra include a central peak (Rayleigh) and two Lorentzian-shaped sidebands (acoustic scattering or Brillouin). The Rayleigh contribution arrives from thermal fluctuations dissipating by thermal conduction. For large ordering parameters, the line width in frequency is given by $D_{T} K^{2}$, where $D_{T}$ is the thermal diffusivity. The Brillouin sidebands are symmetrically Doppler shifted about the central Rayleigh peak due to acoustic scattering from small pressure fluctuations in the gas (propagating at the speed of sound). They are located at $\pm K a / 2 \pi$, and their line width is given by $\Gamma K^{2}$, where $\Gamma$ is the sound attenuation coefficient. Then, the ratio of Rayleigh to Brillouin integrated intensities is $(\gamma-1)$, where $\gamma$ is the ratio of specific heats. ${ }^{13}$ This approximation fails in the kinetic regime when $y$ is negligibly small.

At elevated temperatures for atmospheric pressure (or low pressures at room temperature) $(y<<1)$, if we neglect the acoustic Brillouin scattering, the velocity distribution of molecules is Maxwellian. For one velocity component this is given by

$$
f(\zeta)=\left(\pi w_{\zeta}\right)^{-1 / 2} \exp \left(-(\zeta-v)^{2} / w_{\zeta}^{2}\right)
$$

where $\zeta$ is the velocity of a molecule, $v$ is the bulk velocity of molecules, 


$$
w_{\zeta}=\left(2 k_{B} T / m\right)^{1 / 2}
$$

is the full width at $1 / \mathrm{e}$ height of distribution $\mathrm{f}(\zeta), T$ is the gas temperature, $k_{\mathrm{B}}$ is the Boltzmann constant, and $m$ is the molecular mass. The bulk velocity $v$ is what we observe at macroscopic scale. For a gas at rest the bulk velocity is zero and the average molecular random velocity $u=(\zeta-v)$ due to deviations of $\zeta$ from $v$ is also zero.

Photons of frequency $v$ from the incident probing beam are scattered from molecules moving in all directions with the random velocity $u$, and are shifted in frequency according to the Doppler effect

$$
d v=\left(v-v_{0}\right)=(\mathbf{K} \bullet \mathbf{u}) / 2 \pi
$$

Therefore, for a component of velocity along the resultant wave vector $\mathbf{K}$ one can relate the velocity distribution of the molecules to the scattered light frequency by rewriting Eq. (2) as

$$
f(v)=(\pi w)^{-1 / 2} \exp \left(-(d v / w)^{2}\right),
$$

where the new spectral width in frequency is

$$
w=\left(2 k_{B} T / m\right)^{1 / 2} \sin (\theta / 2) / \lambda_{0} .
$$

In a gas mixture (as for example in air or in a flame) the spectral distribution of the scattered light is a sum of distribution with contributions from each molecular species $i$ weighted by their molar fraction $\chi_{i}$, and the differential scattering cross-section $\sigma_{i}$. Therefore one can write

$$
I(v)=C I_{0} N \sum_{i=1}^{N_{i}} \chi_{i} \sigma_{i} f_{i}(v),
$$

where $I_{0}$ is the laser intensity, and $N$ is the total gas number density. The constant of proportionality $C$ depends on the laser polarization, and wavelength; the size of the probe volume; and the solid angle of the light collection optics (collecting power), the collection direction, and the detector quantum efficiency.

To determine the gas properties, the scattered light spectra and the laser spectra are analyzed with theoretical models by least-square fitting techniques using the laser spectra as reference for Doppler shift calculations [References 7, 10,11, and 12]. Research efforts to extract the properties of gases by analyzing the spectra of scattered light with Fabry-Perot interferometers include in diluted gases,${ }^{13}$ in low-speed combustion flames, ${ }^{14,15,16}$ and in high-speed rocket external flows. ${ }^{17}$ The use of kinetic models to obtain spectral information requires prior knowledge of gas parameters (that are not measured by the instrument) such as the internal specific heat per molecule, the ratio of shear to bulk viscosity, and the ratio of shear viscosity to thermal conductivity. ${ }^{18}$ This model is difficult to use since these parameters are not precisely known and there is no data for the species and range of temperatures required for measurements. In this work we use a simplified Gaussian-Lorentzian analytical model, easy to apply to the data, instead of a more detailed computational Rayleigh-Brillouin kinetic model. Then, corrections of these measured quantities via calibrations are applied to obtain quantitative information within an acceptable margin of error as it will be shown later.

\section{Apparatus}

The novel instrument design is based on the idea of referencing all measurable properties, the velocity, the temperature, and the gas density. ${ }^{19}$ This is performed with an optical setup that simultaneously records the spectral properties of Rayleigh scattered light from two largely separated measurement locations, one of which is used for reference. To increase the incident laser energy probing the measurement volume, and implicitly to increase the scattered signal, this system uses for probing two collinear laser beams slightly delayed in time for probing the gas sample. The laser source is a seeded dual Nd:YAG laser, with a wavelength, $\lambda_{0}$, of $532 \mathrm{~nm}$, variable timing between

pulses, $10 \mathrm{~Hz}$ repetition rate, nine nanoseconds pulse width, and $0.005 \mathrm{~cm}^{-1}$ bandwidth. Referring to Fig. 1, the probing laser beam (of wave vector $\mathbf{k}_{0}$ ) is focused at the measurement volume (mv1) by the $\mathrm{L}_{1}$ lens and collected and re-collimated for further use by the $\mathrm{L}_{2}$ lens. The elastically scattered light pulse (exp1) (of wave vector $\mathbf{k}_{\mathrm{s}}$ ) is collected and collimated from the measurement volume mv1 by the $\mathrm{L}_{3}$ lens $(f / 4,250-\mathrm{mm}$ focal length, located 250 


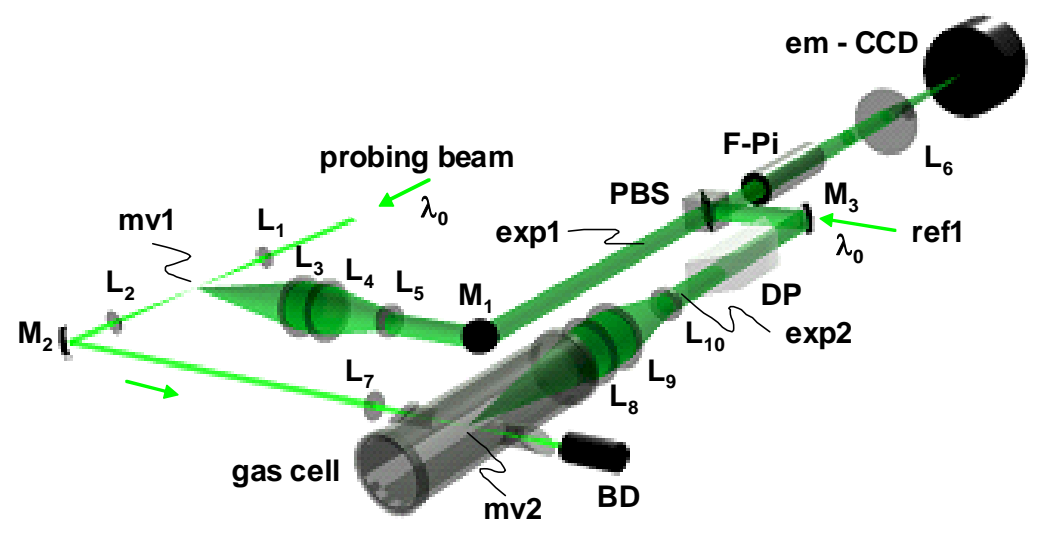

Figure 1. Instrument configuration for simultaneous multi-point measurement of velocity, the gas translational temperature, and density at two largely-separated spatial locations. Legend: $L$ - lens; M planar mirror; BD - beam dump; PBS - polarization dependent beam combiner; DP - Dove prism; F-Pi solid etalon; em-CCD - electron avalanche multiplication CCD camera; mv - measurement volume.

$\mathrm{mm}$ from mv1). The beam diameter of this signal is reduced to match the analyzing optics aperture diameter $(\sim 15 \mathrm{~mm})$ using the beam reducer/expander formed by lenses $\mathrm{L}_{4}$ and $\mathrm{L}_{5}$, and directed using the mirror $\mathrm{M}_{1}$ toward the polarization dependent beam combiner PBS. The beam combiner PBS facilitates the optical mixing of the signal with the un-scattered light (ref1) used for frequency referencing. In this respect, this configuration is similar to previous ones. ${ }^{7}$ In the new configuration, however, the polarization dependent beam combiner PBS is used to also combine this beam with a second beam (exp2) collected by the $\mathrm{L}_{8}$ lens from elastic scattering at a secondary measurement volume mv2. This measurement volume is situated a gas cell with known gas properties that is used for properties referencing as will be shown later. Similarly, this beam is reduced and collimated by the lenses $\mathrm{L}_{9}$ and $\mathrm{L}_{10}$, and directed by mirror $\mathrm{M}_{3}$ toward the combiner PBS. The light polarization is adjusted such that the PBS reflects it together with the main beam toward the Fabry-Perot interferometer (F-Pi). The reference laser light needed for frequency referencing is optically mixed with the signals at the PBS by passing un-shifted laser light through the mirror $\mathrm{M}_{3}$ (or mirror $\mathrm{M}_{1}$ ). Therefore the interferogram formed by the F-Pi and imaged by the $\mathrm{L}_{6}$ lens on the $\mathrm{CCD}$ camera contains the spectra of

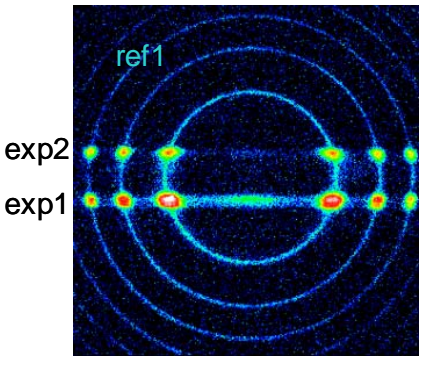

(a)

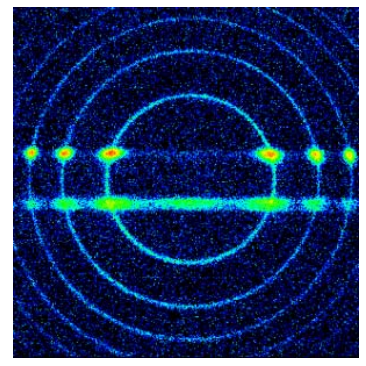

(b)

Figure 2. Fabry-Perot interferograms containing multiple spectra of elastically scattered light from two largely separated measurement locations, one of which is used for reference. The bottom horizontal pattern (exp1) is the signal obtained at the location of a $\mathrm{H}_{2}$-air Hencken burner flame: (a), in air at $293 \mathrm{~K}$ outside the flame, and (b), in the flame at about $1560 \mathrm{~K}$. The top horizontal pattern (exp2) is the signal from the secondary measurement location situated in a lowpressure $\mathbf{N}_{2}$ gas cell at room temperature. This signal is used to reference the measurement of temperature and density performed at the flame location. The concentric ring pattern (ref1) is the spectra of unshifted laser light used to reference the Doppler shift frequency of all signals and facilitate computation of the gas bulk velocity at both locations. exp1, exp2, and ref1. Figure 2 shows examples of interferograms containing multiple Rayleigh signals (exp1 and exp2) obtained in the cell (top horizontal pattern) and at the flame location (bottom horizontal pattern), in room air (293 K in Fig. 2(a)) before flame ignition, and in an $\mathrm{H}_{2}$-air flame at equivalence ratio $\phi=0.5$ (1560 K in Fig. 2(b)), respectively. The circular pattern is the interferogram of the laser light used for frequency reference (ref1). To avoid the eventual overlap of the closely spaced spectra exp1 and exp2 the pattern of exp2 could also be rotated in the image plane (for example by $90^{\circ}$ ) using the Dove prism DP.

As mentioned earlier, in this new arrangement we increase the energy deposited at the measurement volumes by spatially overlapping the two collinear laser beams slightly delayed in time. Additionally we introduce optical aberrations in the optical path of the focusing lens through lens tilting. This beam shaping is achieved by tilting the focusing lens $\mathrm{L}_{1}$ slightly, $5^{\circ}$ to $22^{\circ}$ depending on the laser energy, and thereby introducing an astigmatism aberration

4 
of the beams at their foci. ${ }^{20}$ The tilting is performed about an axis perpendicular to the plane containing the incident laser beam and the signal collection direction. The elliptical beam allows more energy to be deposited in the flow without gas breakdown. This results in larger Rayleigh signal intensity if collected from a direction in the plane of the beam and the major axis of the ellipse, at the cost of reduced spatial resolution in the other dimension. In the experiments reported here a total of $385 \mathrm{~mJ}$ in two laser pulses separated in time by less than 200 nanoseconds and a ratio of major to minor axis of the elliptical beam of about 10 at its first focus were used. The measurement volume is about $0.05 \mathrm{~mm} \times 0.2 \mathrm{~mm} \times 0.5 \mathrm{~mm}$.

Both $\mathrm{L}_{3}$ and $\mathrm{L}_{8}$ lenses collect scattered light from the measurement locations at an angle $\theta=90^{\circ}$ with respect to the corresponding probing laser beam direction. The interferometer is made of solid glass with a free spectral range (FSR) of $8 \mathrm{GHz}$ and a total finesse of about 22. This defines the maximum measurable Doppler shift and therefore the dynamic range for measuring velocities to about $\pm 1.5 \mathrm{~km} / \mathrm{s}$ when no prior velocity direction is known, or to about $3 \mathrm{~km} / \mathrm{s}$ if the velocity direction is known. The range of measurable temperatures and densities in gases is from cryogenic temperatures to about $2500 \mathrm{~K}$ (limited at high temperatures by the overlap of consecutive orders of interference), and from $0.1 \mathrm{~kg} / \mathrm{m}^{3}$ to about $2 \mathrm{~kg} / \mathrm{m}^{3}$ (limited by the CCD dynamic range), respectively.

\section{Experimental}

Data are obtained from a laboratory combustion experiment. The first measurement location is situated in a nonpremixed near-adiabatic $\mathrm{H}_{2}$-air Hencken burner flame (also used to calibrate the CARS measurements ${ }^{9}$ ) at atmospheric pressure. The burner is placed vertically at the first measurement volume (mv1) in a direction perpendicular to the scattering plane. The hydrogen fuel and the air oxidizer are assumed to mix immediately above the burner surface. The burner surface is a $25.4 \mathrm{~mm} \times 25.4 \mathrm{~mm}$ rectangular section of hypodermic needle tubes for fuel and honeycomb passages for oxidant surrounded by a rectangular region of co-flow honeycomb design (a total of $36.5 \mathrm{~mm} \times 36.5 \mathrm{~mm}$ square surface). The $\mathrm{H}_{2}$ and air equivalence ratio $\phi$ is varied from 0.1 to 1 , by varying the flow rates (up to $0.5 \mathrm{l} / \mathrm{sec}$ ). A constant flow of $\mathrm{N}_{2}$ of $0.4 \mathrm{l} / \mathrm{sec}$ was directed through the co-flow nozzle at all fuel-air mixtures to improve flame stability and provide an almost dust-free environment for the instrument. The molecular content in the co-flow-flame interface is considered unknown. The flame temperature and species molar fractions in the flame region were calculated from the adiabatic flame theory. ${ }^{21}$ The primary species are $\mathrm{N}_{2}, \mathrm{O}_{2}, \mathrm{H}_{2} \mathrm{O}$, and $\mathrm{H}_{2}$, with the minor species (Ar, $\mathrm{CO}, \mathrm{OH}$, etc.) being neglected. The product of combustion $\left(\mathrm{H}_{2} \mathrm{O}\right)$ is in the gaseous state with negligible contribution from condensate. The perfect-gas law for the mixture is $p / \rho=(\mathcal{R} / m) T$, where $m$ is the molecular weight of the mixture $(\mathrm{kg} / \mathrm{kmole}), p$ is the gas pressure $\left(\mathrm{N} / \mathrm{m}^{2}\right), \rho$ is the density $\left(\mathrm{kg} / \mathrm{m}^{3}\right)$, and $T$ is the gas temperature $(\mathrm{K})$, and the perfect-gas constant $\mathcal{R}$ is $8314.5 \mathrm{~J} /(\mathrm{kmole})(\mathrm{K})$. The experiments are performed in room air $\left(m_{\text {air }}=28.97 \mathrm{~kg} / \mathrm{kmole}\right)$ at the atmospheric pressure $p_{\text {air }}\left(101324.72 \mathrm{~N} / \mathrm{m}^{2}\right)$, temperature $T_{\text {air }}(293.5 \mathrm{~K})$, and density $\rho_{\text {air }}\left(1.2 \mathrm{~kg} / \mathrm{m}^{3}\right)$. The axial velocity of the flow (vertical direction) was estimated from flow rates at room temperature to about $10 \mathrm{~m} / \mathrm{sec}$, but higher velocities of this order of magnitude are expected during combustion. The radial velocity component (horizontal direction, measured in this experiment) is considered to be much smaller than the axial velocity (near zero).

The second measurement volume is situated in a constant properties glass cell containing $\mathrm{N}_{2}$ at room temperature $(293.5 \mathrm{~K})$. The glass cell is connected with manual valves and plastic tubing to a feedstock of $\mathrm{N}_{2}$ gas and to a vacuum pump. The initial air in the cell is removed with the vacuum pump and the $\mathrm{N}_{2}$ is let in to the desired pressure before measurements. A pressure of $p_{0}=26.5 \mathrm{kPa}$ (198.5 torr) was used for the reported experiments. Lower pressure was used to ensure that the density and the $y$-parameter are similar to those in the combustion flow, which made the signals from both channels comparable.

\section{Results and Discussion}

Verification of the instrument is performed by obtaining all scattered light spectral properties simultaneously in the atmospheric flame and nitrogen $\left(\mathrm{N}_{2}\right)$ cell. Single-shot spectra $I(v)$ obtained in the air and $\mathrm{H}_{2}$-air flame from interferograms following the steps explained in Ref. 7, are shown in Fig. 3. The data are shown with black solid and red empty circles. The best fit to the data shown with red circles using the sum of a Gaussian function for the scattered spectra and a Lorentzian function for the reference laser spectra is shown with a continuous black line. When the reference signal is too low, the properties of the Lorentzian function required for this fit are extracted from 

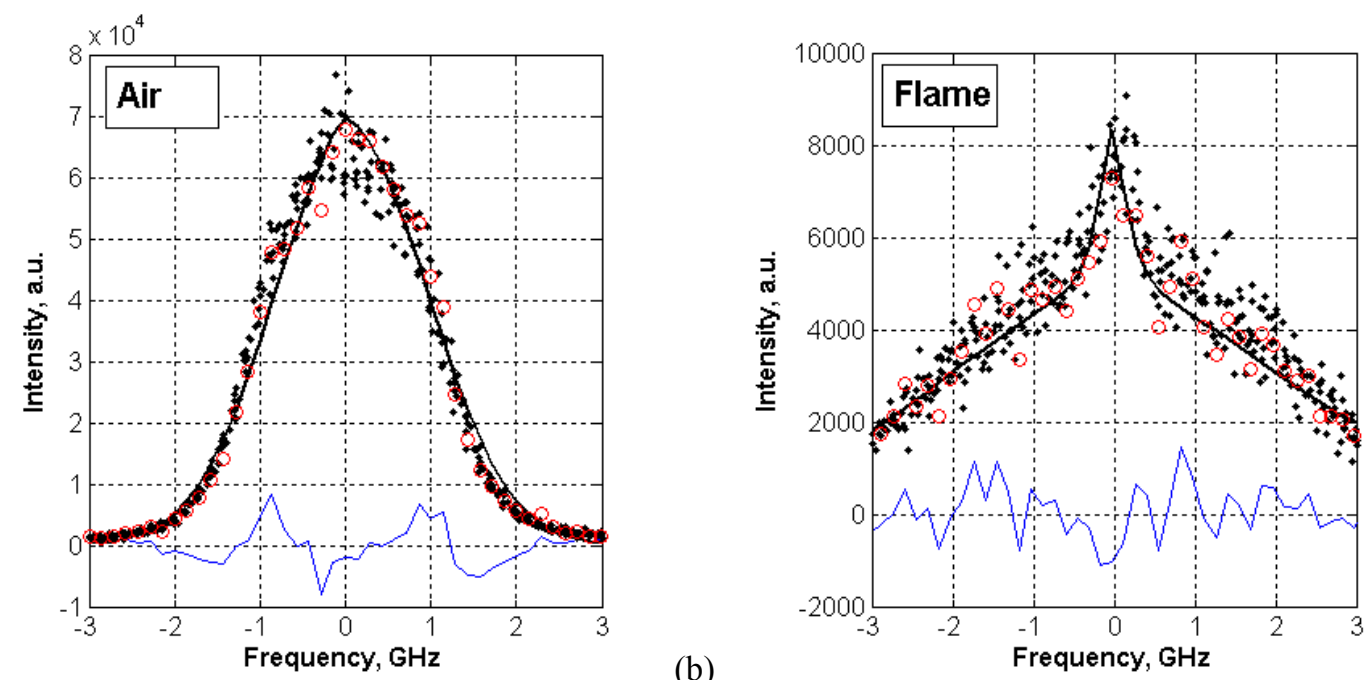

(a)

(b)

Figure 3. Combined Rayleigh and laser spectra obtained, (a), in atmospheric air at $293 \mathrm{~K}$, and (b), in $\mathrm{H}_{2}$-air flame at $\phi$ $\sim 0.5(1637 \mathrm{~K})$. The intensity scale is shown in arbitrary units (a.u.). Data contain ten instantaneous measurements to emphasize the trend (solid circles). Only one dataset (empty red circles) is fit with a combined Gaussian-Lorenzian function (solid black line). The residual of the fit is shown in blue.

the entire reference (circular) pattern that exists outside of the signal pattern (horizontal pattern). This new methodology of finding the location of the reference (Airy function) in the interferogram plane is different from what is described in Ref. 7. A planned future article will describe in detail this algorithm. Ten single-shot spectra are overlapped to the fitted data (solid circles) to emphasize the trend. Figure 3(a), and Fig. 3(b), show the spectra obtained in atmospheric air at the conditions described earlier, and in the flame at $\phi=0.5(\sim 1637 \mathrm{~K})$, respectively. The energy of the laser was maintained approximately constant during these measurements though any variations in laser intensity would be corrected by the data obtained in the reference cell. The parameter $y$ for air at atmospheric conditions is 0.8 so the spectra in Fig. 3(a) should theoretically contain Brillouin sidebands Doppler shifted by about $\pm 0.9 \mathrm{GHz}$ around the Rayleigh center peak. The residual between the experimental data and the Gaussian fit is shown in the plot at the bottom of the figure (blue curve). This indicates that the Gaussian fit function over-predicts the wings and under-predicts the top half of the distribution. Therefore the distinctive maxima of this residual function approximately detect the Brillouin peaks locations and the integral of its absolute value approximately shows the acoustic scattering contribution to the spectra. A theoretical analysis of this integral function of the $y$ parameter, shows that this contribution to the measured Rayleigh spectra is percentwise similar with the one found experimentally by Cattolica et al. ${ }^{14}$ for different gases. We use this relation to correct the measured spectra for the Brillouin contribution at lower gas temperatures.

For a Gaussian profile, the gas translational temperature is calculated from the spectral width. Normalizing Eq.(3) with respect to the known values of properties, the non-dimensional gas translational temperature becomes $T_{m}$ $=W_{m}{ }^{2} m$, where $W_{m}$ is the measured non-dimensional width of the spectral profile, and $m$ the molecular weight of the gas, which is a function of the gas composition, normalized by the molecular weight of air. This relation holds at moderate to high temperatures, but generates large errors without knowing the gas composition, and when the spectral profile deviates from a Gaussian function as described earlier. Since the molecular weight is not measured, it was estimated by comparing the measured width with the computed spectral width, which is a function of the molecular weight of the gas (and temperature). Then, we use the computed molecular weight of the gas as an approximation of the molecular weight of the gas existing at the time of measurement. In doing so we assume that for certain measured spectral widths only a small range of molecular weights are possible. For example, in the calculation of $\boldsymbol{T}_{\mathrm{m}}$ from the spectra shown in Fig. 3, the broadest Rayleigh spectra shown in Fig. 3 (b) cannot be attributed to cold gases (higher molecular weights) that will produce the spectra shown in Fig. 3 (a). The maximum error possible in these computations without considering this approximation of the molecular weight is about $16 \%$ in the whole range of measurable temperatures presented here. The actual error of the measurement obtained by estimating molecular weights is considered to be only a fraction of this maximum error.

To obtain the bulk velocity of the gas, the spectra are analyzed for the average spectral shift frequency relative to the laser spectra. From Eq. 4, if all molecules move with the bulk velocity $v$, the spectra is Doppler shifted from the laser frequency $v_{0}$ by $\left(v-v_{0}\right) / v_{0}=2(v / c) \sin (\theta / 2)$, where $v_{0}\left(=c / \lambda_{0}\right)$ is the frequency reference of the laser. Notice 
that in the previous work described in Refs.14, 15, and 16, a simultaneous frequency reference is not available for every measurement. Such frequency referencing allows for corrections to drifts in the laser frequency and etalon temperature or vibration (in the case of airspaced etalons, though a solid etalon was used in the present experiment).

In a usual configuration for measuring gas density, to keep the measurement unaffected by the fluctuation in laser energy from shot to shot, a reference measurement of the laser energy is implemented by recording a part of the signal or the laser light. The solution for a reference adopted in this work is a secondary measurement volume located in a glass cell containing a gas with known pressure, temperature, and composition. By normalization with the cell signal total intensity $A_{0}$ the laser energy fluctuations from pulse to pulse during the measurement are minimized. Additionally, the measured cell parameters provide simultaneously a reference for known gas density, temperature, and velocity (zero for stagnant gas). Integrating over the solid angle of the collecting optics and rewriting in dimensionless quantities, Eq. (7) becomes $\rho_{m}=C_{2}\left(A_{\mathrm{m}} / A_{0}\right) /\left(\sigma / m_{m}\right)$, where $A_{\mathrm{m}} / A_{0}$ is the non-dimensional integral of
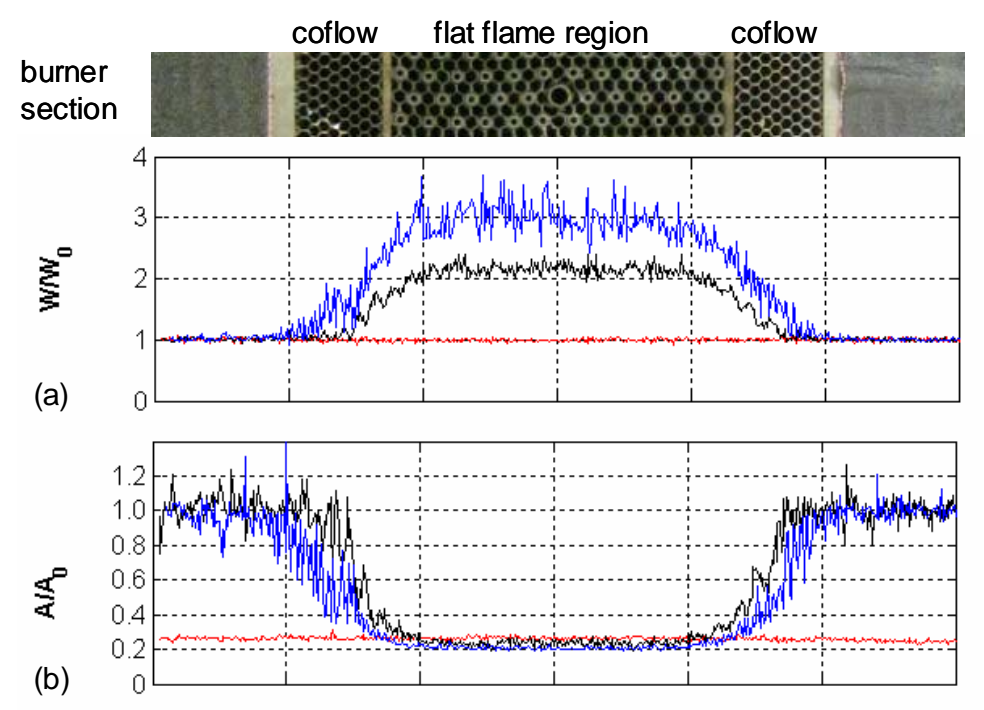

(b)

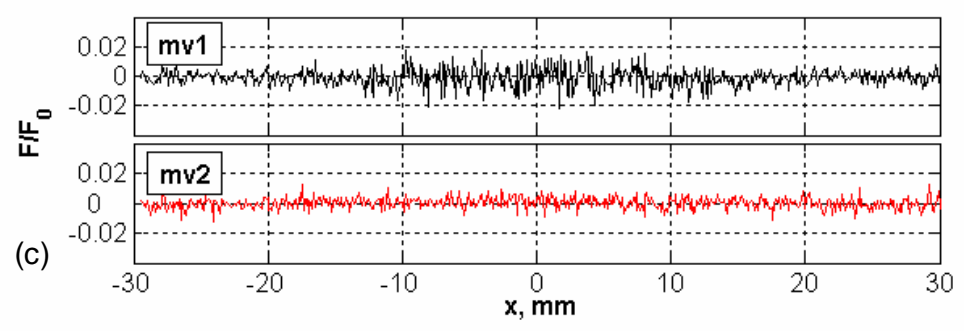

Figure 4. Normalized spectral properties obtained simultaneously in $\mathbf{H}_{2^{-}}$ air Hencken burner flame ( $\phi \sim 1.0$ (blue), and $\phi \sim 0.5$ (black)), and in the reference $\mathrm{N}_{2}$-cell (red symbols) relative to atmospheric properties: (a), the spectral bandwidth, (b), the area of the spectra, and (c), the Doppler shift frequency. The inset on the top of the figure is a top view of the central section of the burner.

the signal, $\rho_{m}$ is the gas density normalized by $\rho_{\text {air }}$, and $\sigma=\Sigma_{i}^{N i}\left(\chi_{i} \sigma_{i}\right) / \sigma_{\text {air }}$ is the non-dimensional scattering cross section of the gas weighted by the molar fraction of species $\chi_{i}$. The constant $C_{2}$ is found from simultaneous measurements of known gas properties in the cell $\left(\mathrm{N}_{2}\right)$ and in air at the measurement volume.

The total measured signal is computed by integrating the spectral fit function (solid line in Fig. 3 minus the reference Lorentzian function). For $y<<1$, the integral can be approximated by (assuming a Gaussian function) the maximum spectral intensity multiplied by full-width half maximum and $(2 \pi)^{1 / 2}$.

Figure 4 shows the profiles of measured quantities in the Hencken burner flame at equivalence ratio of $\phi=1.0$ (blue) and $\phi=0.5$ (black), relative to the known quantities. Data is extracted from single-shot interferograms using Gaussian and Lorentzian fit functions as approximation for the Rayleigh and laser spectra. Figure 4(a) shows the normalized spectral bandwidth $W_{m}$ measured at the flame location. The normalization factor is the spectral width $W_{0}$ obtained at this location in air at room temperature $(293.5 \mathrm{~K})$. The width obtained in the $\mathrm{N}_{2}$ cell, about $9 \%$ narrower than the width measured in the air, can also be used for reference. Figure 4(b) shows the integral of the signal intensity measured at the flame location (black and blue trace), relative to the integral of the signal obtained in the cell, corrected for the cell signal intensity relative to the flame signal intensity. The core flame cross section, where the flame properties are almost uniform, is about $20 \mathrm{~mm} \times 20 \mathrm{~mm}$. The measurement volume was scanned along a line from one side to the other over the center of the burner (shown in the inset image of Fig. 4) in a plane situated at about $38.5 \mathrm{~mm}$ above the burner surface (similar measurements of rotational-vibrational temperature using CARS were performed by Hancock ${ }^{22}$ ). The Doppler shift frequency obtained in flame at equivalence ratio of $\phi$ $=0.5$ (black) relative to the maximum measurable frequency (defined by the interferometer) is shown in Fig. 4 (c). The simultaneous properties measured in the reference cell are plotted with red symbols. The normalization factors for the cell properties in Fig. 4(a) and Fig. 4(b) are the measured cell properties, the spectral width and the integral of the signal in the cell at one atmosphere, respectively. The spatially resolved measurements of the relative spectral width show a slight flame asymmetry in this property as the measurement volume is scanned across the flame region 
(Fig. 4(a)). This is evidenced more at higher flame temperatures (blue) than at intermediate ones (black) although in both cases they correlate approximately with the position of the burner nozzles revealing the discrete (flamelet) structure of the flame. In the core region of the flame, where the ideal flow properties should be predictable from computations, the measured average temperature is $2323 \mathrm{~K} \pm 415 \mathrm{~K}$ ( \pm one standard deviation) on the left side and $2083 \mathrm{~K} \pm 343 \mathrm{~K}$ on the right side of the axis of flame at $\phi=1.0$. These average values are about $2.2 \%$ and $12.4 \%$, respectively, lower than predicted. The large standard deviation of the measurement is attributed partly to the variation with position (during the scan) in flame temperature and composition due to the flamelet structure and partly to random measurement error. At moderate temperatures $(\sim \phi=0.5)$, when the flame is more uniform, this variation is smaller than $80 \mathrm{~K}$. Because the measurements were not performed at a fixed location in the flame, the instrument random error in measuring temperature is not directly quantifiable at this time.

The non-dimensional integral of the signal, which is proportional to the gas density (and inversely proportional to the gas temperature), is shown in Fig. 4(b). The measured density in air, and flame at $\phi=1$ are $1.23 \mathrm{~kg} / \mathrm{m}^{3} \pm 0.05 \mathrm{~kg} / \mathrm{m}^{3}$ and $0.13 \mathrm{~kg} / \mathrm{m}^{3} \pm 0.007 \mathrm{~kg} / \mathrm{m}^{3}$, respectively. These mean values are higher than predicted, by about $2.5 \%$ in room air and by about 4.5 $\%$ at the flame. The higher-than-expected value of density in the flame suggests also a flame of lower temperature than predicted as well as measured from the spectral width.

The gas density, and bulk velocity computed from spectral properties obtained in air and the flame (data shown with black symbols in Fig. 4) during the full scan of the flame at $\phi=0.5$, are shown in Fig. 5 as a function of the computed temperature. The gas density and temperature are normalized with respect to the ambient air density $\left(1.2 \mathrm{~kg} / \mathrm{m}^{3}\right)$ and temperature (293.5 K), respectively. The velocity is normalized by the maximum measurable velocity $(3 \mathrm{~km} / \mathrm{s})$ defined by the optical setup. As shown in Fig. 5(a), the measured gas density dependence on the measured translational temperature (blue symbols), agrees very well with the perfect gas law (solid curve) computed for this case. In this computation the gas temperature and composition is assumed to be only a function of $\phi$ so the gas molecular weight can be inferred as a function of the temperature outside of the flame core where $\phi$ is unknown. The measured velocity dependence on the flame temperature is shown in Fig. 5(b). The rms (root mean squre) variations that include true innate instrument errors and spatial and temporal fluctuations of the flow As expected, the mean velocity is near zero, and the rms fluctuations ( \pm one standard deviation) of velocity increases with increasing temperature from about $11 \mathrm{~m} / \mathrm{s}$ at the room temperature (and in the cell) to about $27 \mathrm{~m} / \mathrm{s}$ in the flame core (and increases to $39 \mathrm{~m} / \mathrm{s}$ at $\phi=1$ ). This is less than $1 \%$ of the dynamic range of the instrument. We must mention that this instrument was not intended to measure near-zero velocities, but rather supersonic/hypersonic ones (up to $3 \mathrm{~km} / \mathrm{s}$ !). To accurately measure low velocity flows, a higher resolution interferometer and a backward scattering direction (more sensitive to Doppler shift) could be selected.

To our knowledge, these high resolution measurements of simultaneous and instantaneous gas translational temperature, velocity, and density using interferometric Rayleigh scattering are the first to be performed in $\mathrm{H}_{2}$-air Hencken burner flames. 


\section{Conclusions}

A non-intrusive optical diagnostic technique for temporally and spatially resolved measurements of multiple properties in reacting and non-reacting gases is described. The system employs a narrow-band pulsed laser source at $532 \mathrm{~nm}$ for simultaneous probing of molecules through elastic light scattering at two largely separated measurement locations, one of which is used for reference. The collected photons containing information from both measurement locations are analyzed spectrally with a planar Fabry-Perot interferometer. The reference measurement is located in a glass cell containing $\mathrm{N}_{2}$ gas at low pressure $(26.5 \mathrm{kPa})$ and room temperature $(293.5 \mathrm{~K})$. The measured cell spectral properties provide simultaneously a reference for known gas density (the spectral intensity), translational temperature (the spectral width), and supplementary near-zero gas velocity (zero average Doppler shift). Additionally, the cell spectral intensity provides the normalization factor for the laser energy fluctuations critical for the measurement of density. These spectral properties are used to calculate, by normalization, the gas density and translational temperature at the measurement location. The range of measurable temperatures and densities in gases is from cryogenic temperatures to about $2500 \mathrm{~K}$ (limited by the interferometer), and from $0.1 \mathrm{~kg} / \mathrm{m}^{3}$ to about $2 \mathrm{~kg} / \mathrm{m}^{3}$ (limited by the CCD dynamic range), respectively. The maximum dynamic range for measuring velocities is about $\pm 1.5 \mathrm{~km} / \mathrm{s}$ when no prior velocity direction is known, or about $3 \mathrm{~km} / \mathrm{s}$ if the velocity direction is predictable.

In a laboratory demonstration, the measurement location is situated in a non-premixed near-adiabatic $\mathrm{H}_{2}$-air Hencken burner flame at atmospheric pressure. The referenced measurements at different flame conditions compare favorably with the flame temperature and the gas density (up to the stoichiometric flame temperature of $2377 \mathrm{~K}$ ) computed with the adiabatic flame theory. The random errors of measuring the flame properties, the translational temperature, velocity, and the gas density at the maximum measurable gas temperature, are estimated to less than about $17 \%, 39 \mathrm{~m} / \mathrm{s}$ and $5 \%$, respectively. We must emphasize that these errors include the spatial and temporal variation of these properties across the flame.

These preliminary experiments demonstrate simultaneous referencing (calibration) while performing measurements of velocity, density, and temperature with the interferometric Rayleigh scattering technique described here. Future improvements include calibration tests and the use of better theoretical models to extract the information more accurately from scattered light spectra. Envisioned is the use of the instrument for simultaneous multi-property turbulence studies of subsonic, supersonic, and hypersonic, nonreacting flows and predictable composition reacting flows in the range of temperatures, turbulence levels, and errors described here.

\section{Acknowledgments}

This work was supported by the NASA Fundamental Aeronautics Program - Hypersonics Project, Experimental Capabilities and Propulsion Disciplines. We would like to acknowledge Greg Herring and Robert Baurle for many helpful discussions, and Gaetano Magnotti and Lloyd Wilson for laboratory setup and operation of the Hencken Burner flame.

\section{References}

${ }^{1}$ Drummond, J. P., Danehy, P. M., Bivolaru, D., Gaffney, Jr. R. L., Tedder, S. A., and Cutler, A. D., "Supersonic combustion research at NASA, 2007 Fall Technical Meeting," Eastern States Section of the Combustion Institute, University of Virginia, October 21-24, 2007; Also, Drummond, J. P., et al., "Development of Methods to Predict the Effects of Test Media in GroundBased Propulsion Testing," NASA/TM-2009-215766, 2009.

${ }^{2}$ Baurle, R. A., "Modeling of High Speed Reacting Flows: Established Practices and Future Challenges," AIAA-2004-0267, 42th Aerospace Sciences Meeting, Reno, NV, January 5-8, 2004.

${ }^{3}$ Cutler, A. D., Magnotti, G., Baurle, R. A., Bivolaru, D., Tedder, S. A., Danehy, P. M., Weikl, M. C., Beyrau, F., and Seeger, T., "Development of Supersonic Combustion Experiments for CFD Modeling", AIAA Paper 2007-0978, 45th AIAA Aerospace Sciences Meeting and Exhibit, Reno, Nevada, January 8-11 (2007).

${ }^{4}$ Farassat, F., Doty, M. J., and Hunter, C. A., "The Acoustic Analogy - A Powerful Tool in Aeroacoustics with Emphasis on Jet Noise Prediction," AIAA Paper No. 2004-2872, Presented at 10th AIAA/CEAS Aeroacoustics Conference, Manchester, United Kingdom, May 10-12, 2004.

${ }^{5}$ Eckbreth, A., "Laser Diagnostics for Combustion Temperature and Species." Combustion Science and technology Series, Vol. 3, $2^{\text {nd }}$ ed., Gordon \& Breach Publishers, Taylor \& Francis Books, Inc., New York, NY, 1996.

${ }^{6}$ Miles, R. B., Lempert, W. R., and Forkey, J. N., "Laser Rayleigh Scattering," Measurement Science and Technology, Vol. 12, 2001, pp. 33-51.

${ }^{7}$ Bivolaru, D., Danehy, P. M., Gaffney, Jr. R. L., and Cutler, A. D., "Direct-View Multi-Point Two-Component Interferometric Rayleigh Scattering Velocimeter," AIAA-2008-0236, 46th Aerospace Sciences Meeting, Reno, NV, January 9-12, 2008.

${ }^{8}$ O'Byrne, S., Danehy, P. M., Cutler, A. D., Tedder, S. A., "Dual-Pump Coherent Anti-Stokes Raman Scattering Measurements in a Supersonic Combustor," AIAA Journal, Vol. 45, No. 4, 2007, pp. 922-933. 
9 Danehy, P. M., Magnotti, G., Bivolaru, D., Tedder, S., and Cutler, A. D., "Simultaneous Temperature and Velocity Measurements in a Large-scale, Supersonic, Heated Jet," Paper 1193, 55th JANNAF Propulsion Meeting, Boston, MA, May 1216, 2008. Also, D. Bivolaru, P.M. Danehy, and A. D. Cutler, "Simultaneous CARS and Interferometric Rayleigh Scattering," Review of Scientific instruments (to be published).

${ }^{10}$ Bivolaru, D., Lee, J. W., Jones, S. B., Tedder, S., Danehy, P. M., Weikl, M. C., Magnotti, G., and Cutler, A. D., "Mobile Rayleigh - CARS Instrument for Simultaneous Spectroscopic Measurement of Multiple Properties in Gaseous Flows," 22nd International Congress on Instrumentation in Aerospace Simulations Facilities (ICIASF), Monterey, CA, June, 2007. See also, Bivolaru, D, Danehy, P.M., Grinstead, K.D., Jr., Tedder, S., and Cutler, A.D., "Simultaneous CARS and Interferometric Rayleigh Scattering," AIAA-2006-2968, 25th AIAA Aerodynamic Measurement Technology and Ground Testing Conference, San Francisco, CA, June 5-8, 2006.

${ }^{11}$ Bivolaru, D., Danehy, P. M., Lee, J. W., "Interferometric Rayleigh Scattering Measurement System,” NASA, Washington, DC, U.S. Patent No. 7414708, August 18, 2008.

${ }^{12}$ Bivolaru, D. Danehy, P. M., Cutler, A. D., "Multipoint Inteferometric Rayleigh Scattering using Light Recirculation," Paper Number 2008-3708, 26th AIAA Aerodynamic Measurement Technology and Ground Testing Conference, Seattle, WA June 2326, 2008.

${ }^{13}$ Yip, S. J., "Rayleigh Scattering from Diluted Gases,” Acoust. Soc. Am. 49, 941-949 (1971).

${ }^{14}$ Cattolica, R., Robeen,, and Talbot, L., "The Interpretation of Spectral Structure of Rayleigh Scattered light from Combustion Gases," AIAA-1976-0031, 14th Aerospace Sciences Meeting,Washington, DC, January 26-28. 1976.

15 Pitz, R. W., Cattolica, R., Robben, F., Talbot, L., "Temperature and Density in Hydrogen-Air Flame From Rayleigh Scattering," Combustion and Flame, 27, 313-320 (1976).

${ }^{16}$ Dibble, R. W., Starner, S. H., Masry, A. R., and Barlow, R. S., "An Improved Method of Data Acquisition and Reduction for Laser Raman-Rayleigh and Fluorescence Scattering from Multispecies,” Appl. Phys. B, Vol. 51, No. 1, 1990, pp. 39-43.

${ }^{17}$ Seasholtz, R. G. , Zupanc, F. J., and Schneider, S. J., "Spectrally resolved Rayleigh scattering diagnostic for hydrogen-oxygen rocket plume studies,” J. Propulsion Power, Vol 8, No. 5, 1992, pp. 935-942.

${ }^{18}$ Young, A. T., and Kattawar, G. W., "Rayleigh Scattering Line Profiles," Applied Optics, Vol. 22, No. 23, 1983, pp. 36683670 .

${ }^{19}$ Bivolaru, D., Danehy, P. M., and Cutler, A. D.,, "Dual-cavity Rayleigh Scattering Measurement System," The George Washington University, Washington, DC, U. S. Provisional patent application, No. 61/346599, May 20, 2010.

${ }^{20}$ Matthews, S. C., "Tilted Lens Imager in a Laser Amplifier/Oscillator and Method Utilizing Same," Hughes Aircraft Co., los Angeles, CA, U.S. Patent No. 5228051, July 13, 1993.

${ }^{21}$ Gordon, S., and McBride, B. J., "Computer Program for Calculation of Complex Chemical Equilibrium Compositions, Rocket Performance, Incident and Reflected Shocks, and Chapman-Jouguet Detonations," NASA Report: SP-273, 1976.

22 Hancock, R. D., Bertagnolli, K. E., Lucht, R. P., "Nitrogen and Hydrogen CARS Temperature Measurements in a Hydrogen/Air Flame Using a Near-Adiabatic Flat-Flame Burner,” Combustion and Flame, Vol. 109, No. 3, 1997 , pp. $323-331$. 